\title{
Treatment of Cutaneous Leishmaniasis with Photodynamic Therapy: The First Case Report from Khyber Pakhthunkhwa (KPK), Swat Pakistan
}

\author{
Khan $\mathrm{A}^{1}$, Khan $\mathrm{K}^{1,2 *}$, Biradar $\mathbf{G}^{1}$, Ali $\mathrm{A}^{1}$ and Khan $\mathbf{M}^{3}$ \\ ${ }^{1}$ Swat Institute of Nuclear Medicine and Radiotherapy (SINOR), Swat, Pakistan \\ ${ }^{2}$ Department of Physics, University of Malakand, Pakistan \\ ${ }^{3}$ Peshawar Medical College, Peshawar KPK, Pakistan
}

*Corresponding author: Karim Khan, Swat Institute of Nuclear Medicine and Radiotherapy

\section{Case Report \\ Volume 5 Issue 1}

Received Date: January 29, 2020

Published Date: February 27, 2020

DOI: $10.23880 /$ cdoaj-16000203 (SINOR), Swat, KPK, Pakistan, Tel: +929469240422, Fax: +9294671160; Email: Karimkhanmph@yahoo.com

\section{Abstract}

Background: Cutaneous Leishmaniasis (CL), the most common subtype of Leishmaniasis, is a vector borne disease transmitted by sand fly.

Case Presentation: Herein, we report a case of CL and, for the first time, its treatment with photodynamic therapy (PDT) in KPK, Pakistan. Specifically, a 55-year-old female patient presented with a single chronic disfiguring ulcerated lesion on the chin. The patient had received pentavalent antimoniate for the last 2 years, with no satisfactory outcome. We treated the patient with methylaminolevulinate based PDT and observed complete response after three session of the PDT treatment, each with a light energy dose of $75 \mathrm{~J} / \mathrm{cm} 2$.

Conclusion: The patient has remained disease-free for over 2 years. This study demonstrates the promising role of PDT in the treatment of CL.

Keywords: Cutaneous leishmaniasis; Photodynamic therapy; Pentavalent antimoniate; Photosensitizer; Methylaminolevulinate

\section{Introduction}

Leishmaniasis, the sixth most important disease affecting the public health worldwide, is a vector borne disease, transmitted among mammalian hosts by female sand fly [1], primarily affecting the skin. Many animal species can carry Leishmania parasite including rodents, dogs and foxes [2]. Rarely Leishmanisis can be passed from person to person through blood transfusion infected needles and syringes or congenitally from mother to baby. The disease can be diagnosed by performing blood test for antibodies against parasite or by urine tests, Polymerase chain reaction (PCR) test can identify parasite based on its genetic fingerprint [3]. The best test to identify the parasite is documenting its presence through a microscope (smear test).

There are several types of Leishmaniasis. For instance, cutaneous Leishmaniasis (CL), a relatively benign skin condition, is characterized by sore(s) on the skin, which usually develop in few weeks after the bite of the sand fly [4]. The sores may heal spontaneously within 12-24 months if the immunity of the patient is strong enough. World Health 
Organization (WHO) estimates 1-1.5 million new cases of CL every year [5]. In Pakistan, the incident of CL is increasing, most likely due to poverty, inadequate medical facilities, negligible planning for vector control, armed conflicts and mass migration of human and cattle. Mucocutaneous Leishmaniasis (MCL) is a less common form, either carried by direct bite of sand fly on mucus membranes or extension to mucosa membranes from skin lesion. It can lead to partial or complete destruction of mucous membranes in the nose, throat or mouth. Visceral Leishmaniasis (VL) is the systemic form of the disease, incubation period may range from days to year after the bite of infected sand fly, and it affects the internal organ usually the spleen, liver, and bone marrow. People symptomatically present with episodes of fever, weight loss, anemia, and enlargement of the spleen and liver [4].

The conventional treatment of CL is directed toward eradication of amastigotes and reduction of the size of the lesions to promote healing. At present meglumine antimoniate and sodium stibogluconate are first line drugs worldwide [6,7]. Photodynamic therapy (PDT) is a rapidly evolving therapeutic option for CL. The FDA approved indications of PDT include actinic keratosis, basal cell carcinoma, and superficial squamous cell carcinoma, while inflammatory skin diseases, such as psoriasis, acne vulgaris, and sarcoidosis are the off label uses of PDT. Moreover, PDT have a crucial role in the treatment of infectious skin diseases, including verruca vulgaris, condyloma acuminatum, and CL $[8,9]$.

Herein, we present the first case of CL treated with PDT from Pakistan. Specifically, a 55 year old female patient presented with a CL lesion on the chin, which did not responded to pentavalent antimoniate. The patient showed complete response to PDT, being followed up regularly and has remained disease-free for over 2.5 years

\section{Case Report}

A 55 year old female patient presented with the complaints of a single chronic disfiguring ulcerated lesion on the chin, as shown in Figure 1a. The lesion had been diagnosed as cutaneous Leishmaniasis (CL). The patient had received treatment (i.e., pentavalent antimoniate) for the last 2 years, with no satisfactory outcome. Clinical assessment showed that the patient had developed resistance to pentavalent antimoniate. The patient was planned for photodynamic therapy (PDT). Written and informed consent was obtained from the patient.

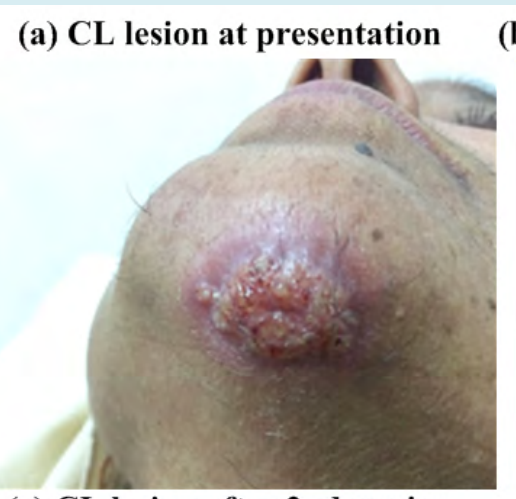

(c) CL lesion after 2nd session

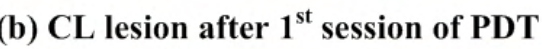
of PDT

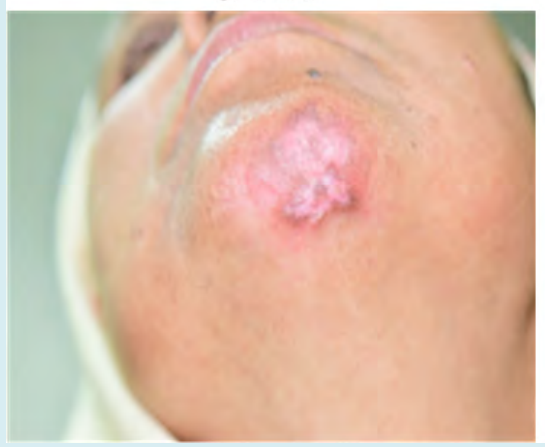

(d) CL lesion after 3rd session of PDT

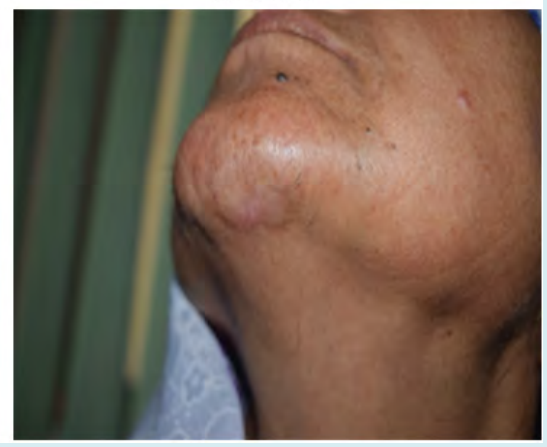

Figure 1: Sample white light photos summarizing the progress of PDT treatment of the CL lesion. Single chronic disfiguring ulcerated lesion on the chin (a), express significant improvement after first (b) and second (c) session of PDT. Complete response with excellent cosmetic results was observed after third session of PDT (d). 


\section{Clinical Dermatology Open Access Journal}

To perform PDT, the lesion and the adjacent skin were cleaned and scrubbed to remove the necrotic layer and exudates dried up. The photosensitizer, methylaminolevulinate (MAL), was applied locally on the lesion under adhesive covering, followed by an incubation period of 3 hours for absorption of the photosensitizer. After incubation period, the cream was removed and the lesion was washed with normal saline. The lesion was irradiated with red laser light (wavelength $635 \mathrm{~nm}$ ); a light energy dose of $75 \mathrm{~J} / \mathrm{cm} 2$ was delivered, as per the institutional protocol. The patient received three sessions of PDT; the first two sessions were repeated at two weeks interval, while the third session was given at one month interval. The clinical features of the patient, lesion characteristics and PDT protocol used have been summarized in Table 1 .

\begin{tabular}{|c|c|c|c|c|c|}
\hline \multicolumn{2}{|c|}{ Clinical Features } & \multicolumn{2}{c|}{ Lesion Characteristics } & \multicolumn{2}{c|}{ PDT Treatment } \\
\hline Age & 55 years & Site & Chin & Photosensitizer & MAL* $^{*}$ \\
\hline Gender & Female & Size & $4 \mathrm{~cm}$ diameter & Laser wavelength & $635 \mathrm{~nm}$ \\
\hline Previous Treatment & Pentavalent antimoniate & Ulceration & Present & Light dose & $75 \mathrm{~J} / \mathrm{cm} 2$ \\
\hline Treatment outcome & No response & Disfigurement & Present & No. of sessions & 3 \\
\hline
\end{tabular}

Table 1: Summary of clinical features of the patient with CL, lesion characteristics and PDT.

The response of the PDT was evaluated through clinical examination, and evaluation of inflammation was assessed by erythema and swelling and size of lesion. Figure $1 \mathrm{~b}-\mathrm{d}$ shows the progressive improvement of the patient. Currently, the patient is on regular follow up and remained disease free after over two years. Besides the complete response, excellent cosmetic outcome with no recurrence throughout the observation time and no complication was observed.

\section{Discussion}

The incidence/ prevalence of Leishmaniasis is alarming in Pakistan [1], presumably due to low socio-economic conditions and lack of awareness and education. The situation is even exacerbating by the limited advancement at the therapeutic end, as there is no significant improvement in the treatment of CL for moSre than 90 years worldwide. The Leishmania species has been reported to developed resistance to the previaling drugs $[10,11]$; in some cases, the resistance is as high as $65 \%$. Moreover, the prevailing treatment paradigm of the CL has been reported to cause higher rate of side effects, which include renal, hepatic and cardiac toxicity, myalgia, joint stiffness, malaise, anorexia and bradycardia with ECG changes, prolongation of the QT interval and T-wave inversion [12]. Apart from potential systemic toxicities, these medicines have limited success of complete response rates and a healing period of over 4 months [13]. In addition, the current treatment protocol necessitates time investment for daily clinical visits (for the subcutaneous injections) and patient discomfort. In summary, despite high incidence Leishmaniasis in Pakistan, cumbersome treatment protocols and limited success in conventional therapy, it remains the first choice of treatment for Leishmaniasis $[14,15]$.

Photodynamic therapy (PDT) may offer an improved alternate treatment for Leishmaniasis, potentially countering the shortcomings of the prevailing treatment(31), as it allows for lower treatment costs, no or limited profile of side effects and higher treatment efficacy. Specifically, Leishmaniasis has been shown to exhibit complete response in about $95 \%$ and partial response in about 5\% of the patients with just 3-4 treatment sessions of PDT. Moreover, it has markedly reduced the healing time to less than three months. In addition, the PDT treatment has no known side effects except for mild burning sensation and pain during the application of laser light only; however, these are well-tolerated and manageable.

In this study, we have successfully treated a case of cutaneous Leishmaniasis, which did not responded to the prolonged treatment (i.e., two years) of pentavalent antimoniate. PDT not only reduced the treatment time significantly (i.e., from over 02 years to 1.5 months), but also resulted in elevated efficacy. Moreover, the patient ease and satisfaction for PDT was markedly high, as the treatment offered excellent cosmetic outcome without any considerable side effect. Consequent upon the encouraging results from the treatment of the case, we have planned (and initiated) a regional project for the treatment of Leishmaniasis with PDT (a manuscript based on the initial results is in the preparation phase).

\section{Conclusion}

We presented, for the first time, the successful treatment of cutaneous Leishmaniasis (CL) with photodynamic therapy (PDT). Specifically, three sessions of PDT relieved the systemic symptoms (i.e., erythema, swelling and progressive reduction in size of the lesion) of CL and the patient was disease-free for more than 2 years of follow-up. Moreover, the PDT treatment offered excellent cosmetic results. This study demonstrates that CL respond promisingly to PDT 


\section{Clinical Dermatology Open Access Journal}

treatment.

\section{References}

1. Durrani AZ, Durrani HZ, Kamal N (2012) Prevalence of Leishmania in sand fly in Pakistan. Pak J Zool 44(1): 6165.

2. Durrani AZ, Durrani HZ, Kamal N, Mehmood N (2011) Prevalence of cutaneous Leishmaniasis in humans and dogs in Pakistan. Pak J Zool 43(2): 263-271.

3. Cruz I, Millet A, Carrillo E, Chenik M, Salotra P, et al. (2013) An approach for interlaboratory comparison of conventional and real-time PCR assays for diagnosis of human Leishmaniasis. Exp Parasitol 134(3): 281-289.

4. Gawade S, Nanaware M, Gokhale RM, Adhav PS (2012) Visceral Leishmaniasis: a case report. Australas Med J 5(2): 130-134.

5. World Health Organization (2015) Investing to overcome the global impact of neglected tropical diseases: third WHO report on neglected diseases. World Health Organization, Geneva.

6. Herwaldt BL, Berman JD (1992) Recommendations for treating Leishmaniasis with sodium stibogluconate (Pentostam) and review of pertinent clinical studies. Am J Trop Med Hyg 46(3): 296-306.

7. Al-Jaser M, El-Yazigi A, Croft SL (1995) Pharmacokinetics of antimony in patients treated with sodium stibogluconate for cutaneous Leishmaniasis. Pharm Res 12(1): 113-116.

8. MacCormack MA (2008) Photodynamic therapy in dermatology: an update on applications and outcomes. Semin Cutan Med Surg 27(1): 52-62.

9. Gardlo K, Horska Z, David CE, Rauch L, Megahed M, et al. (2003) Treatment of cutaneous Leishmaniasis by photodynamic therapy. J Am Acad Dermatol 48(6): 893896.

10. Sundar S, More DK, Singh MK, Singh VP, Sharma S, et al. (2000) Failure of pentavalent antimony in visceral Leishmaniasis in India: report from the center of the Indian epidemic. Clin Infect Dis 31(4): 1104-1107.

11. Sundar S, Thakur BB, Tandon AK, Agrawal NR, Mishra CP, et al. (1994) Clinico-epidemiological study of drug resistance in Indian kala-azar. BMJ 308(6924): 307.

12. Thakur CP, Kumar M, Kumar P, Mishra BN, Pandey AK (1988) Rationalisation of regimens of treatment of kalaazar with sodium stibogluconate in India: a randomised study. Br Med J (Clin Res Ed) 296: 1557-1561.

13. Thakur CP, Kumar M, Pandey AK (1991) Evaluation of efficacy of longer durations of therapy of fresh cases of kala-azar with sodium stibogluconate. Indian J Med Res 93: 103-110.

14. Jha TK, Singh NK, Jha SN (1992) Therapeutic use of sodium stibogluconate in kala-azar from some hyperendemic districts of N Bihar, India (Abstr). J Assoc Physicians India 40: 868.

15. Sundar S, Singh VP, Sharma S, Makharia MK, Murray HW (1997) Response to interferon-ó plus pentavalent antimony in Indian visceral Leishmaniasis. J Infect Dis 176(4): 1117-1119. 